\title{
Original Article \\ Is there a positive effect of participation on a clinical trial for patients with advanced non- small cell lung cancer?
}

\author{
Rajappa S, Gundeti S, Uppalapati S, Jiwatani S, Abhyankar A, Pal C, Digumarti R \\ Department of Medical Oncology and Pathology, Nizam's Institute of Medical Sciences, Punjagutta, \\ Hyderabad-500 016, India
}

Correspondence to: Dr. Senthil Rajappa, E-mail: siddharth142@sify.com

\begin{abstract}
BACKGROUND: There is general belief that patients who enrolled on a clinical trial have better outcomes compared to those who are treated outside of a trial. We analyzed if there was a 'trial effect' for patients with advanced non-small cell lung cancer (NSCLC) treated with chemotherapy. MATERIALS AND METHODS: A retrospective analysis of cohorts of patients with advanced NSCLC who received chemotherapy inside and outside of a clinical trial were analyzed for response rates (RR), progression free survival (PFS), overall survival (OS), 1 and 2 year survival. RESULTS: There were 194 patients who received chemotherapy of which, 54 were on a clinical trial and 140 outside of it. For the whole group, the RR, median PFS, OS, one and two-year survivals were $35.4 \%$, six months (range, 2-70), seven months (range, $2-72$ ), $29.8 \%$ and $9.7 \%$ respectively. The differences in RR and PFS of patients who were treated inside and outside of a clinical trial were not significant $(P=0.6164,0.0881)$. The differences in median $O S$ and one-year survivals between the groups were significant $(P=0.0052,0.022)$. For the whole group, patients who received II line chemotherapy had better OS $(P \leq 0.0001)$. More patients in the trial group received II line chemotherapy $(P=0.0004)$. The difference in the median OS between the groups continued to be significant even after patients who received II line chemotherapy were censored $(P=0.0437)$. CONCLUSION: Patients with advanced NSCLC who were treated inside of a clinical trial had better OS compared to those who were treated outside of it.
\end{abstract}

Key words: Chemotherapy, experimental arm effect, lung cancer, survival, trial effect

\section{Introduction}

There has been a phenomenal increase in the number of cancer clinical trials conducted in India. ${ }^{[1]}$ In the era of evidence based medicine, it is extremely important to conduct clinical trials, especially Phase III trials, to get valid scientific answers to questions pertaining to therapy. A part from this, the other major benefit from a patient's point of view, especially in a health care system where there is no managed care, is that patients are guaranteed of therapy without the associated financial burden.

Many oncologists believe that patients with cancer who enroll in a clinical trial have better outcomes than those who did not enroll. ${ }^{[2]}$ The American Society of Clinical $\mathrm{O}$ ncology and the $\mathrm{N}$ ational $\mathrm{C}$ omprehensive $\mathrm{C}$ ancer $\mathrm{N}$ etwork recommend that the best way to manage

158 a cancer patient is enrollment on a clinical trial. ${ }^{[3,4]}$ $\mathrm{H}$ owever, there has been lack of high quality scientific evidence to support this belief and recommendation.

There is evidence for and against the view that enrollment into clinical trials lead to better outcomes. $^{[5-7]}$ H owever, showing a causal relationship between trial participation and improved outcome, referred to as 'the trial effect', is difficult. ${ }^{[2]} \mathrm{O}$ ur department has been actively involved in the conduct of Phase I-IV clinical trials for the past 15 years. Nonsmall cell lung cancer (NSCLC) is one area where we have been highly successful in the recruitment of patients.

In order to assess, if the outcomes of patients with advanced NSCLC who were treated inside of a clinical trial were different from those treated outside of it, 
we studied the response rates (RR), progression free survival (PFS), overall survival (OS), one and two-year survival of these two groups of patients.

\section{Materials and Methods}

$D$ ata from medical records of patients with untreated stage IIIB and IV NSCLC who received I line platinum based doublet chemotherapy inside and outside of a clinical trial during the years 2002-2006 were collected.

The diagnosis of NSCLC was confirmed either by fine needle aspiration or a biopsy. The staging investigations included a contrast enhanced computed tomography scan (CECT) of the chest and upper abdomen. A bone scan and CECT scan of the brain were done where appropriate. $O$ ther investigations before therapy included complete blood counts, liver and renal function tests. Other biochemical tests were done wherever indicated.

The institutional ethics committee and scientific review board approved all the clinical trials for patients with stage III B and IV NSCLC and an informed consent was given by all patients before entry on to a clinical trial. Patients who were treated outside of a clinical trial were treated with various regimens administered intravenously ranging from I generation platinum based doublets like Cisplatinum $\left(75 \mathrm{mg} / \mathrm{m}^{2}\right) /$ carboplatin (AU C 5) Day 1 + etoposide (100 mg/m² Days 1-3) (EP) or II generation regimens like gemcitabine $(1250 \mathrm{mg} /$ $\mathrm{m}^{2}$ Day 1 and 8$)+$ cisplatinum $\left(75 \mathrm{mg} / \mathrm{m}^{2}\right.$ Day 1$) /$ carboplatin (AU C 5 Day1) (GC) and Paclitaxel (175 $\mathrm{mg} / \mathrm{m}^{2}$ Day 1$)+$ cisplatinum $\left(75 \mathrm{mg} / \mathrm{m}^{2}\right.$ Day 1$) /$ carboplatin (AU C 5 Day1) (TC). Patients were also given radiotherapy either as definitive loco regional therapy in stage III B or with palliative intent for the primary or metastatic sites in stage IV. These patients were treated outside of a clinical trial for various reasons: lack of willingness to sign the informed consent refusing randomization, fear of adverse events due to a new drug, unsure of efficacy of the experimental arm, unsure if they would be able to comply with the rigorous follow-up requirements in a trial or if they had III party reimbursement.

All patients inside a clinical trial were those from our hospital who participated in various randomized controlled trials conducted as part of a global multi centre studies. They were part of the following studies: a) Pemetrexed + Cisplatin vs Gemcitabine + Cisplatin [all patients on this trial were taken for this analysis, published Scagliotti et al, JCO 2008; Vol 26: No 21], no difference in efficacy between the arms] b) Paclitaxel + Carboplatin + human recombinant lactoferrin vs
Paclitaxel + Carboplatin [JCO 2006 ASCO Proceedings Part I June 20 Supplement, Vol 24, No 18S: 7095, a small study of 100 patients where response rate was the primary objective, $\mathrm{P}$ values for PFS and OS not reported, final publication not done] c) Paclitaxel + Carboplatin + oral thrombopoietin vs Paclitaxel + Carboplatin [unpublished, all patients from this trial were taken for analysis, Thrombopoietin, a drug that was expected to prevent thrombocytopenia, is not expected to improve efficacy of chemotherapy]. Patients treated inside of a clinical trial received these drugs in the same schedules and doses as those treated outside of a trial. No patients outside of a trial were treated with Pemetrexed while those on a trial received the drug as follows: Pemetrexed $\left(500 / \mathrm{m}^{2}\right)+$ Cisplatinum $\left(75 \mathrm{mg} / \mathrm{m}^{2}\right)$, both given as IV infusion on Day 1 of 3 weekly cycle. Routine prophylaxis with dexamethasone, vitamin B12 and folic acid were used.

Patients were given a maximum of six cycles of chemotherapy. Response evaluation was performed after every two to three cycles of chemotherapy by clinical examination and CECT chest and upper abdomen. $\mathrm{N}$ one received any maintenance chemotherapy. At progression patients were administered II line chemotherapy with either Inj. D ocetaxel $75 \mathrm{mg} / \mathrm{m}^{2}$ IV D ay 1 every 21 days or Cap. Gefitinib $250 \mathrm{mg}$ daily until progression. No patients in this analysis were enrolled on clinical trials for II line chemotherapy.

The following response criteria were used:

A complete response (CR) was defined as disappearance of all the lesions on radiology. Partial response (PR) was defined as a decrease of $30 \%$ in the sum of the longest diameters of all target lesions. Stable disease (SD) was defined as patients who neither did nor fit into either partial response or progressive disease. Progressive disease (PD) was defined as an increase of $20 \%$ in the sum of the longest diameters of the target lesions or appearance of a new lesion at any time during or after therapy.

The outcome parameters were response rate $(R R)$, progression free survival (PFS), overall survival (OS), one-year and two-year survivals.

Progression free survival (PFS) was defined as the time from start of chemotherapy to the time that progressive disease was documented, death or lost for follow-up. O verall survival was defined as the time from start of chemotherapy to death due to any cause.

U nivariate and multivariate analysis was done to assess the effect of age, sex, smoking status, performance status, stage and treatment with a I or II generation 
platinum doublet on overall survival. Patients were also compared for all outcome parameters with respect to whether they were treated within or out of a clinical trial.

\section{Statistical methods}

GraphPad software Quick Cals online calculator was used to calculate the $P$ values for the categorical and continuous variables. For continuous variables, the $P$ value was calculated using the unpaired test to compare the means. For categorical data like stage, smoking, sex, performance status and response rates, the 2-tailed $\mathrm{P}$ value was calculated using Fisher's exact test and $2 \times 2$ contingency table.

Graph Pad Prism software for windows Version 4, 2003 was used to plot the Kaplan Meier curves for PFS and OS. U nivariate analysis for OS was done by plotting $K$ aplan Meier curves and the log rank test was used to calculate $P$ values. Logistic regression analysis for multivariate analysis for OS was carried out using $M$ et calc. demo version statistical software using the same independent variables after coding. A $P$ value $<0.05$ was considered as statistically significant.

\section{Results}

Between the years 2002-2006, 194 patients received chemotherapy for stage III B and IV NSCLC of which 54 patients were on a clinical trial and 140 were treated out of a trial. The median age of patients was 58 years (range, 16-88) with a male: female ratio of $4: 1$. These patients were evaluated for outcome parametersRR, median PFS, OS, one and two-year survival. The baseline characteristics of all patients are in Table 1.

The outcome parameters for the whole group of 194 patients is summarized in Table 2. There were 69 patients who had a CR or PR amounting to a RR of $35.4 \%$. The median PFS, OS, one and two-year survivals were six months (range 2-70), seven months (range $2-72$ ), $29.8 \%$ and $9.7 \%$ respectively.

The RR of $40 \%$ for patients treated on a clinical trial was not significantly different from the $34.2 \%$ for patients treated out of a trial $(P=0.7564)$ [Table 3].

U nivariate analysis was performed for age ( $<50$ vs $>50$ years), gender (male vs female), smoking status (yes vs no), stage (IIIB vs IV), performance status (PS, 0-1 vs 2 ) and treatment regimens (I vs a II generation platinum doublet) for overall survival.

On univariate analysis, the strongest predictors for overall survival were female gender, absence of history

\begin{tabular}{|c|c|}
\hline Character & N (\%) \\
\hline Age in years (median) & $58(16-88)$ \\
\hline Males & $59(16-88)$ \\
\hline Females & $53(18-87)$ \\
\hline Sex ratio & $4: 1$ \\
\hline Males & $155(80)$ \\
\hline Females & $39(20)$ \\
\hline \multicolumn{2}{|l|}{ ECOG Performance status } \\
\hline$<2$ & $126(65)$ \\
\hline$>2$ & $68(35)$ \\
\hline \multicolumn{2}{|l|}{ Smoking/tobacco use } \\
\hline Males & $124 / 155(80)$ \\
\hline Females & 2/39 (5) \\
\hline \multicolumn{2}{|l|}{ Stage } \\
\hline IIIB & $112(58)$ \\
\hline IV & $84(42)$ \\
\hline \multicolumn{2}{|l|}{ I line chemotherapy } \\
\hline On a clinical trial & $54(28)$ \\
\hline Out of a clinical trial & $140(72)$ \\
\hline
\end{tabular}

\section{Table 2: Treatment outcomes for all patients} ( $n=194)$

\begin{tabular}{lc} 
Parameter & N (\%) \\
\hline Complete response & $6(3.0)$ \\
\hline Partial response & $63(32.4)$ \\
\hline Stable disease & $61(31.4)$ \\
\hline Progressive disease & $64(32.9)$ \\
\hline PFS (months) & $6(2-70)$ \\
\hline OS (months) & $7(2-72)$ \\
\hline 1 year OS (\%) & $58 / 194(29.8)$ \\
\hline 2 year OS (\%) & $19 / 194(9.7)$ \\
\hline PFS-Progression free survival, OS-Overall survival &
\end{tabular}

of smoking and PS $(P=0.0057,0.0013,0.0074)$. Age, stage, and treatment regimens did not predict significantly for overall survival $(P=0.2758,0.2556$, $0.8353)$. On multivariate analysis, only $P S(P=0.0387)$ was significant.

\section{Characteristics and outcomes for patients treated on a clinical trial versus out of a clinical trial}

The baseline clinical characteristics and outcome measures of patients who were treated in and out 


\begin{tabular}{lcc}
\hline Table 3: Response rates & & \\
\hline Response & $\begin{array}{c}\text { Trials } \\
(\mathbf{n}=\mathbf{5 4})\end{array}$ & $\begin{array}{c}\text { Non trials } \\
(\mathbf{n}=\mathbf{1 4 0})\end{array}$ \\
\hline Complete response (\%) & $2(3.7)$ & $4(2.8)$ \\
\hline Partial response (\%) & $19(35.2)$ & $44(31.4)$ \\
\hline Stable disease (\%) & $18(33.4)$ & $43(30.7)$ \\
\hline Progressive disease (\%) & $15(27.7)$ & $49(35.1)$ \\
\hline$P=0.7564$ & &
\end{tabular}

of a clinical trial were compared [Table 4]. Both the groups were matched for age, gender, smoking status, performance status and stage $(P=0.3,0.27,0.62,0.18$ and0.74). The mean number of first line chemotherapy cycles in the trial group was 4.6 compared to 4.3 cycles in the non-trial group $(P=0.272)$.

The difference in the response rates and median progression free survival were not significant $(P=0.75$ and 0.08) [Figure 1]. However, the median overall survival of patients treated on a clinical trial was superior at 9.5 months compared to seven months for those patients who were treated out of a trial $(P=0.0052)$ [Figure 2]. The one-year overall survival in the clinical trial group was $42.5 \%$ compared to $25 \%$ on the non-trial group $(P=0.022)$. H owever, the difference in the two-year survivals ( $14.8 \%$ vs $7.8 \%)$ was not significant $(P=0.17)$.

\begin{tabular}{|c|c|c|c|}
\hline & $\begin{array}{l}\text { Trials } \\
(n=54)\end{array}$ & $\begin{array}{l}\text { Non-trials } \\
\text { (140) }\end{array}$ & $\begin{array}{c}\text { Significance } \\
P \text { value }\end{array}$ \\
\hline Age (range) & $55.5(18-82)$ & $53.5(27-85)$ & 0.307 \\
\hline \multirow[t]{2}{*}{ Gender (\%) } & $\mathrm{F}: 17$ (31.5) & $F: 33(23.5)$ & 0.27 \\
\hline & M: 37 (68.5) & M: 107 (76.5) & \\
\hline Smoking (\%) & $34(63)$ & $81(57.8)$ & 0.6251 \\
\hline \multirow[t]{2}{*}{ Stage (\%) } & IIIB: 24 (44.4) & IIIB: 67 (48) & 0.7488 \\
\hline & IV: 30 (55.6) & IV: 73 (52) & \\
\hline \multicolumn{4}{|l|}{ PS } \\
\hline 0 and 1 & $39(72.7)$ & $85(60.7)$ & 0.1818 \\
\hline 2 and 3 & $15(27.3)$ & 55 (39.3) & \\
\hline \multicolumn{4}{|l|}{ Response rate } \\
\hline$(\mathrm{CR}+\mathrm{PR}) \%$ & $21(40)$ & $48(34.2)$ & 0.6164 \\
\hline PFS(months) & $7(2-70)$ & $6(2-33)$ & 0.0881 \\
\hline OS(months) & $9.5(2-72)$ & $7(3-42)$ & 0.0052 \\
\hline 1 year OS (\%) & $23(42.5)$ & $35(25)$ & 0.022 \\
\hline 2 year OS (\%) & $8(14.8)$ & $11(7.8)$ & 0.1776 \\
\hline
\end{tabular}

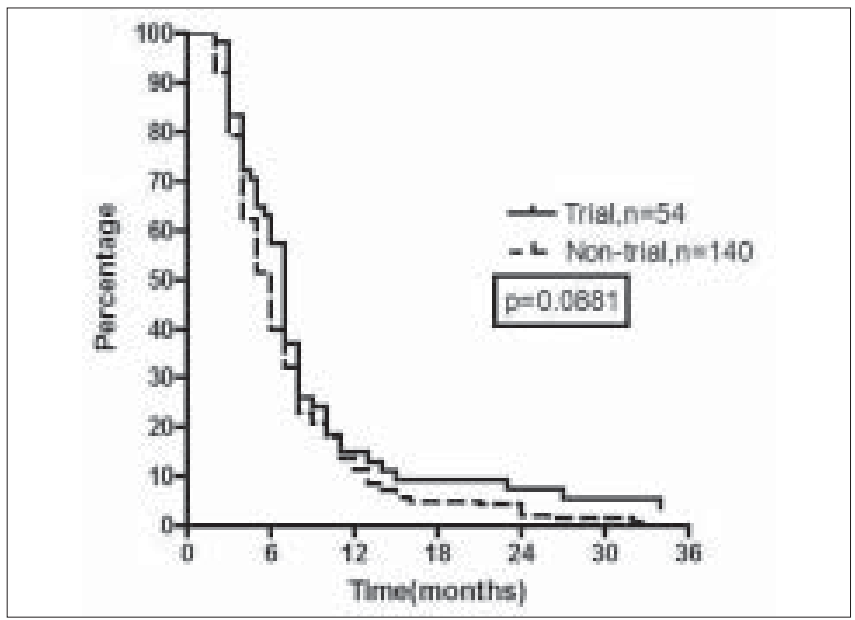

Figure 1: Kaplan Meier estimates of progression free survival

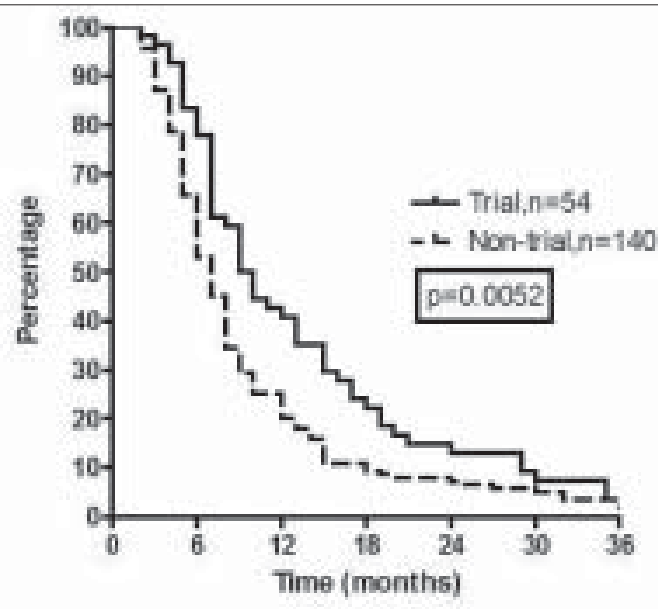

Figure 2: Kaplan Meier estimates of overall survival

O ut of 194, 92(47.4\%) patients received radiation therapy for loco regional control or at distant sites for palliation. Twenty two patients $(40.7 \%)$ of the trial group and 70 patients $(50 \%)$ from the non trial group received $R T(P=0.148)$.

Of the 194 patients, $40(20.6 \%)$ took second line chemotherapy with D ocetaxel or G efitinib. The overall survivals of patients who took II line chemotherapy was 15 months compared to seven months those who did not receive it $(P=<0.0001)$.

Of 54 patients who were on a clinical trial, 18 (33.4\%) took II line chemotherapy compared to 20 of 140 (15.7\%) patients who were treated out of a clinical trial $(P=0.0004)$. When these 38 patients who received II line chemotherapy were only analyzed, the difference in median OS (15.7 vs 12 months, $P=0.6481$ ) [Figure 3] was similar irrespective of whether they received I line chemotherapy on or off a trial.

In order to determine if the better OS of patients 
on a clinical trial was due to the effect of II line chemotherapy or a true clinical trial effect, the survival analysis was done after censoring patients who received II line chemotherapy in both groups. After censoring, the difference in the median OS (7 vs 6 months) was still significant $(P=0.0437)$ [Figure 4].

\section{Discussion}

There is a general belief among physicians that patients enrolled on clinical trials have better outcomes than those who are treated on a regular basis with the standard of care for that particular disease and stage. In an analysis of 26 comparisons from 24 different articles on whether patients on a clinical trial have better outcomes, the conclusion was that there is insufficient data that a trial effect exists. ${ }^{[2]}$ Similarly, the Cochrane review also concludes that the outcomes of patients treated inside of a trial are not different from those treated outside of $i^{1} .{ }^{[8]} \mathrm{H}$ ence, we compared the outcomes for those patients who were enrolled on a

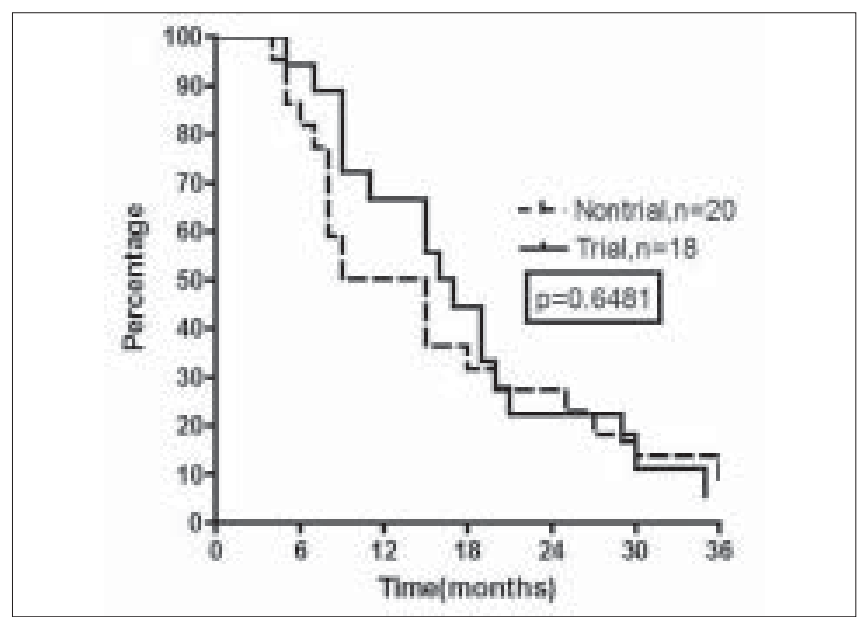

Figure 3: Kaplan Meier estimates of overall survival for patients who received II line chemotherapy

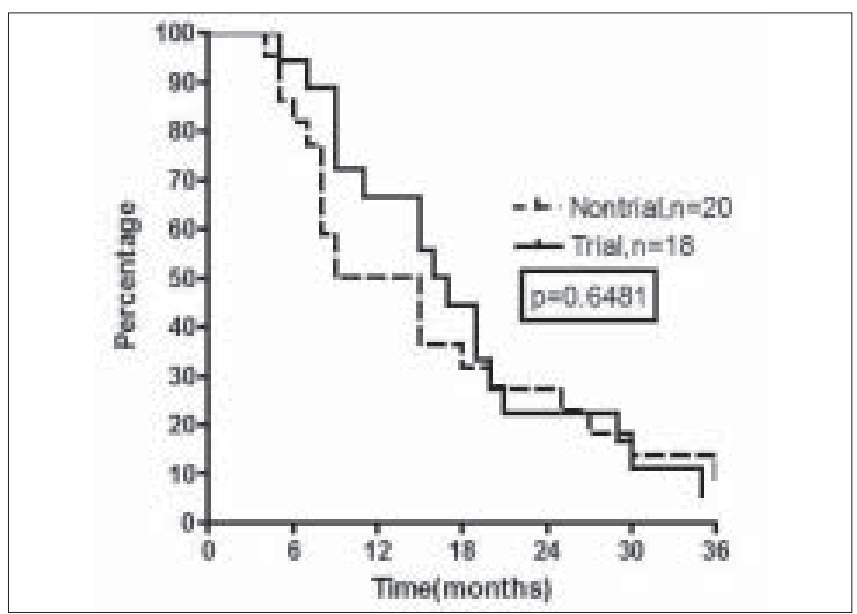

Figure 4: Kaplan Meier estimates for survival for patients who did not receive II line chemotherapy clinical trial for stage IIIB/IV lung cancer to those who were treated out of a trial to look for a 'trial effect'. The difference in the median overall survival and one-year survival was significantly in favor of trial patients.

There are many possible reasons for the better outcomes for patients on a trial. These include an 'experimental treatment effect' where the experimental treatment offered in a clinical trial was superior to the standard therapy and 'participation or trial effect' where trial participation other than exposure to the investigational therapy might cause the improvement (e.g.) the way in which treatments are delivered (protocol effect), incidental aspects of care (care effect) etc. ${ }^{[2]}$ The other reasons may be due to confounding factors like differences in baseline characteristics, the fact that trial participants may belong to a favorable prognostic subset with inherent good outcomes, treatment expertise in centers that conduct these trials, better follow-up with more complete data that is not censored for patients who are lost for followup and publication bias. ${ }^{[2,9-11]}$

In our analysis, patients who received II line chemotherapy at progression had superior outcomes compared to those who did not receive it and significantly more patients who received their I line chemotherapy on a clinical trial went on to receive II line chemotherapy. This seems to be the major reason for the patients in the trial group having superior outcomes. However, we tried to look for a true 'trial effect' and analyzed survival after patients who received II line chemotherapy were censored. The difference in the median survivals continued to be significant denoting a high probability of a 'trial effect' for the improved outcome.

All patients were treated at a single center and both groups were matched for the relevant baseline characteristics. All patients received chemotherapy with platinum based doublets. The results of therapy for advanced NSCLC with the various platinum based doublets are similar. The mean number of cycles and the proportion of patients who received radiotherapy were not different between the groups. Patients in the experimental arms did not receive any other investigational agents that might have contributed to the superior outcomes, ruling out 'experimental treatment effect'.

$\mathrm{H}$ ence, after ruling out most of the other factors that could have contributed to the improved survival of patients enrolled on a clinical trial, we attribute this superior outcome to a 'true clinical trial effect'. However, we cannot rule out the confounding effect of unmatched biochemical and molecular prognostic markers that 
could have led to this outcome. As in our study, most published studies addressing the issue of a clinical trial effect have much smaller numbers on the trial arm compared to the non-trial arm. ${ }^{[2]}$ This reflects the ground reality all over the world, where enrollment into oncology clinical trials is limited to a small proportion of all patients $(<5 \%)$.

It is extremely difficult to objectively assess a clinical trial effect. Most studies that have tried to analyze this effect are retrospective and flawed. ${ }^{[2,12]}$ The best way to do it may be to perform a double randomization where patients are initially assigned to treatment on or out of a trial and the second randomization to a double blind trial for therapy. However, for ethical and logistical reasons such a trial will probably be impossible to do.

In conclusion, patients enrolled on clinical trials for advanced NSCLC have better overall survivals. More patients should be encouraged to enroll on a clinical trial. A part from providing relevant answers to scientific questions, clinical trials, at least in the Indian context, are also likely to provide more patients with therapy free of cost and may possibly be associated with improved outcomes.

\section{References}

1. Raina V. Doing cancer trials in India: Opportunities and pitfalls. Ann Oncol 2005; 16:1567-8.
2. Peppercorn JM, Weeks JC, Cook EF, Joffe S. Comparison of outcomes in cancer patients treated within and outside clinical trials: Conceptual framework and structured review. Lancet 2004;363:263-70.

3. American Society of Clinical Oncology. Available from: http:// www.asco.org.

4. National Comprehensive Cancer Network. Available from: http:// www.nccn.org/professionals/physician_gls/f_guidelines.asp. [last accessed on 2008 Mar 7].

5. Roy P, Vaughan HG, Vaughan HB, Esteve J, Swerdlow AJ. Long term survival in Hodgkins disease patients: A comparison of relative survival in patients in trials and those recorded in population based cancer registries. Eur J Cancer 2000;36:384-9.

6. Braunholtz DA, Edwards SJ, Lilford RJ. Are randomized clinical trials good for us (in the short term)? Evidence for a "trial effect". J Clin Epidemiol 2001;54:217-24.

7. Lantos JD. The "inclusion benefit" in clinical trials. J Pediatr 1999; 134:130-1.

8. Vist GE, Hagen KB, Devereaux PJ, Bryant D, Kristoffersen DT, Oxman AD. Outcomes of patients who participate in randomised controlled trials compared to similar patients receiving similar interventions who do not participate. Cochr Data Met Rev 2005;1:MR000009.

9. Antman K, Amato D, Wood D, Carson J, Suit H, Proppe K, et al. Selection bias in clinical trials. J Clin Oncol 1985;3:1142-7.

10. Schmoor C, Olschewski M, Schumacher M. Randomised and non randomized patients in clinical trials: Experiences with comprehensive cohort studies. Stat Med 1996;15:263-71.

11. Fergusson D, Aaron SD, Guyatt G, Hebert P. Post randomization exclusions: The intention to treat principle and excluding patients from analysis. Br Med J 2002;325:652-4.

12. Burgers JA, Arance A, Ashcroft L, Hodgetts J, Lomax L, Tatcher N. Identical chemotherapy schedules given on and off trial protocol in small cell lung cancer: Responses and survival results. $\mathrm{Br} J$ Cancer 2002;87:562-6

Source of Support: Nil, Conflict of Interest: None declared.

\section{Author Help: Sending a revised article}

1) Include the referees' remarks and point to point clarification to those remarks at the beginning in the revised article file itself. In addition, mark the changes as underlined or coloured text in the article. Please include in a single file

a. referees' comments

b. point to point clarifications on the comments

c. revised article with text highlighting the changes done

2) Include the original comments of the review ers/editor with point to point reply at the beginning of the article in the 'Article File'. To ensure that the reviewer can assess the revised paper in timely fashion, please reply to the comments of the referees/editors in the following manner.

- There is no data on follow -up of these patients.

Authors' Reply: The follow up of patients have been included in the results section [Page 3, para 2]

- Authors should highlight the relation of complication to duration of diabetes.

Authors' Reply: The complications as seen in our study group has been included in the results section [Page 4, Table] 